\title{
Identification and description of Indian parasitic bee genus Sphecodes Latreille 1804, (Halictidae: Hymenoptera)
}

\author{
M. Balaji Rajkumar ${ }^{1 *}$ and Debjani Dey ${ }^{2}$ \\ ${ }^{1 \& 2}$ Division of Entomology, Indian Agricultural Research Institute, New Delhi-110012, INDIA \\ ${ }^{1}$ Current address: Central Institute for Subtropical Horticulture, Lucknow- 226101-(Uttar Pradesh), INDIA \\ *Corresponding author. E-mail: balaento.ento@gmail.com
}

Received: June 12, 2015; Revised received: July 20, 2016; Accepted: October 19, 2016

Abstract: The present study provides an updated knowledge on taxonomy of three important species of Sphecodes Latreille, 1804 which were collected from different parts of India. Three species viz., Sphecodes iridipennis Smith 1879, S. gibbus Smith 1853, S. crassicornis Smith 1879 are redescribed with illustrations, genitalic features and measurements of their morphological features. An annotated checklist of species Sphecodes from India also provided.

Keywords: Cuckoo bee, Halictidae, Hymenoptera Sphecodes, Parasitic bee

\section{INTRODUCTION}

The genus Sphecodes belongs to the family halictidae which are commonly known as 'sweat bees' and distributed widely all over the world. The habitats of halictids are so diverse and they are potential pollinators of more than hundred crops including cereals, fruits, vegetables and medicinal herbs. It is one of the largest bee groups consisting of 3600 described species worldwide. Unlike honey bees, halictid bees lead a solitary or quasi-social life and live in nest or burrows built in the soil or wood. The halictid bees differs from other bee groups by following set of characters viz., basal vein strongly arched in forewing, single subantennal suture, first flagellar segment shorter than scape, lacinia represented by small, hairy lobe on anterior surface of labiomaxillary tube above rest of maxilla and stigma well developed. In total, 211 species were reported so far from India of which 22 species are under genus Sphecodes. The genus Sphecodes are the only parasitic group among the subfamily Halictinae. It has been reported as cleptoparasitic on Halictus, Lasioglossum etc., belonging to its own family and also on other bee families, Andrenidae, Colletidae, Melittidae (Michener, 2007).

The species of genus Sphecodes are usually with red metasoma, wider head, broad clypeus sparsely covered with hairs and the body length ranging from 4.5 to 15 $\mathrm{mm}$ (Michener, 2007). Body slender and appendages without substantial modifications. The sculpture of scutum and propodeum are the unique features amongst the species. Obviously males possess more significant features than female in differentiating species. Hagens (1882) illustrated the male genitalia of Sphecodes for the first time and few other workers contributed remarkably on world species viz., Bluthgen, 1927, Tsuneki, 1983 and Warncke, 1992. The latter author has done regional work on west palaearctic species of genus Sphecodes Latr and provided taxonomic notes and key for identification of 39 species.

The earliest known record of genus Sphecodes from India was in "Catalogue of Insects in the Hymenopterous collections of the British Museum" by Smith (1853). He described two new species viz., fumipennis and apicatus. Later apicatus was synonymised to gibbus by Warncke, 1992. Again during 1879 he described another four new species from India viz., $S$. crassicornis, $S$. albifrons, $S$. irridipennis and $S$. monta$n u s$, all of which are still valid. Bingham (1898) described a new species $S$. indicus which are collected from Shimla and he also described a new species Halictus cameronii, during 1897 later it was transferred to $S$. decorus. Nurse (1903) described six new species from India viz., S. sutor, S. hanuman, S. abuensis, $S$. tantalus $S$. perplexus and $S$. desertus. Three of them are still valid species while other species were later synonymised viz., $S$. sutor with $S$. gibbus, $S$. hanuman with $S$. monilicornis, $S$. desertus with $S$. olivieri by subsequent authors. Bluthgen (1927) did a comprehensive work on Indomalayan bees and described eleven species of Sphecodes including five new species from India and all of which are still valid. He also provided illustrations and key to new species. Cockerell (1911) recorded female of $S$. cameronii (Bingham) and described its body color. All the above 
earlier work provided us the basis for study of Indian Sphecodes. However, the descriptions were sketchy on various morphological features, measurements and illustrations of male genitalia. In particular, there were no detailed descriptions of Indian species S. crassicornis, S. gibbus and S. irridipennis. Keeping this in view, the present study was undertaken to suffice the gap in existing knowledge.

\section{MATERIALS AND METHODS}

The bee specimens from the National Pusa Collection (NPC), Indian Agricultural Research Institute, New Delhi, India as well as collected from different locations of India viz., Madhya Pradesh, New Delhi and Tamil Nadu were studied. The collected specimens were sorted and grouped together with the keys of Bluthgen 1927 and Warncke, 1992. The bees are usually loaded with pollens or spills of nectar on the body, which obscures the morphological characters. So the specimens were washed with tap water followed by mixture of warm water with a drop of liquid soap in $100 \mathrm{ml}$, then again washed with tap water and blotted on tissue paper. The specimens were then dehydrated using $95 \%$ alcohol and sponged down with paper towel until the moisture was removed. Before studying male genitalia, the dry specimens were relaxed in moist chamber containing moist filter papers and phenol. After that the apical segments (from S5) of abdomen were removed carefully and boiled in $10 \%$ potassium hydroxide for 1-2 minutes and then repeatedly washed with distilled water. Finally it was neutralized with glacial acetic acid and dehydrated with $70 \%$ alcohol.
After study it was stored in a small vial contains glycerol for future use. The illustrations and photographs of processed specimens were made using Camera Lucida (Leica 10308700, Leica DFC 425 C), Leica LZ 205 FA. The images were processed further using adobe photoshop.

The standard terminology of Michener (2007) was used for the description of pilosity, sculptures and genitalia etc. the term 'pilosity' used in the description referred to type and arrangement of hairs and 'sculptures' referred to punctures, depressions and patterns on the cuticle. The morphometric details of morphological features viz., body length, head length and width, length of scutum, scutellum, propodeum, wings etc., are given in millimeter or as ratio. The antennal flagellum referred to as F1, F2, F3 etc., following the pedicel. The term apical bands or transverse fascia are used for abdominal tergal bands. The metasomal terga and sterna referred as $\mathrm{T}$ and $\mathrm{S}$ respectively and their numbers given in alphabets viz., T1, S1 etc. The term basal propodeum or propodeal triangle is used for mid dorsum of propodeum. The term outer and inner gonostylus was used if the gonostylus broad or could not viewed from dorsal or ventral. The geographical distribution compiled were based on literature available and many new distribution records within the country are also being reported based on the data labels of specimens actually examined and collections made during the course of study from different places of India. The new records on the distributions of species are asterisked $\left(^{*}\right)$ in the checklist. The checklist includes annotations of each reference.

\section{RESULTS}

Annotated checklist of genus Sphecodes Latreille, 1804 from India (Modified after Ascher and Pickering, 2014)

Subfamily: Halictinae

Genus Sphecodes Latreille, 1804

Sphecodes Latreille, 1804: 182

Dichroa Illiger, 1806: 46

Sabulicola Verhoeff, 1890: 328

Drepanium Robertson, 1903: 103

Proteraner Robertson, 1903: 103

Sphecodium Robertson, 1903: 104

Machaeris Robertson, 1903: 104

Dialonia Robertson, 1903: 104

Sphecodes (Callosphecodes) Friese, 1909: 182.

Type species: Sphex gibba Linnaeus 1785: 571 (monobasic).

\begin{tabular}{cll}
\hline S.N. & Species & Distribution \\
\hline 1 & $\begin{array}{c}\text { abuensis Nurse, 1903: 549 } \\
\text { Sphecodes abuensis Nurse, 1903: 549 }\end{array}$ & Rajasthan: Mount Abu, \\
2 & $\begin{array}{l}\text { albifrons Smith, 1879: 27 } \\
\text { Sphecodes albifrons Smith, 1879: } 27\end{array}$ & Western India \\
3 & $\begin{array}{c}\text { assamensis Blüthgen, 1927 } \\
\text { Sphecodes assamensis Blüthgen, 1927: } 60\end{array}$ & Assam
\end{tabular}


M. Balaji Rajkumar and Debjani Dey / J. Appl. \& Nat. Sci. 8 (4): 1839 - 1849 (2016)

7 dissimilandus (Cameron 1896)

Halictus dissimilandus Cameron 1896: 95

$8 \quad$ fumipennis Smith, 1853

Sphecodes fumipennis Smith, 1853: 36

9

gibbus (Linnaeus, 1785: 571)

Sphex gibba Linnaeus, 1785: 571

Apis glabra Fuebly, 1775: 51

Andrena ferrugena Olivier, 1789: 139

Apis gibbosa Christ, 1791: 177

Melitta sphecoides Kirby, 1802: 46

Melitta picea Kirby, 1802: 48

Andrena austriaca Fabricius, 1804: 325

Sphecodes apicatus Smith, 1853: 36; Warncke, 1992: 29

Sphecodes nippon Meyer, 1922: 171

Sphecodes castilianus Bluthgen, 1924: 473; Warncke, 1992: 29

10 indicus Bingham, 1898

Sphecodes indicus Bingham, 1898: 123

11 invidus (Cameron 1896)

Halictus invidus Cameron 1896:96

12 iridipennis Smith, 1879

Sphecodes iridipennis Smith, 1879: 27

13 lasimensis Blüthgen, 1927

Sphecodes lasimensis Blüthgen, 1927: 40

14 monilicornis (Kirby 1802)

Melitta monilicornis Kirby, 1802:47

Sphecodes maculatus Lepeletier, 1841:545

Sphecodes subquadratus Smith, 1845:1014

Sphecodes gibbus var ephippium subvar rufipes Sichel, 1865:428

Sphecodes gibbus var ephippium subvar dubious Sichel, 1865:419

Sphecodes gibbus var ephippium subvar incertus Sichel, 1865:420

Sphecodes gibbus var ephippium subvar nigrescens Sichel, 1865: 427

Sphecodes gibbus var ephippium subvar testaceipes Sichel, 1865:428

Sphecodes ruficrus Dalla Torre, 1896: 10

Sphecodes hanuman Nurse, 1903:538;

Sphecodes monilicornis var nigerrima Blüthgen, 1927:41;

Sphecodes caucasicus Meyer, 1920:124

Sphecodes monilicornis quadratus Meyer, 1920: 129

Sphecodes monilicornis berberus Warncke, 1992:22

montanus Smith, 1879

Sphecodes montanus Smith, 1879: 27
Bihar: Chapra

West Bengal

West Bengal: Kolkata

*Tamil Nadu: Coimbatore

\section{Uttarakhand (Mussoorie)}

Uttarakhand

Sikkim (Rangit valley), Assam

Jammu \& Kashmir: Srinagar

Himachal Pradesh: Simla; Uttarakhand:

Mussoorie

Uttarakhand

*Bihar: Chapra

Himachal Pradesh: Simla

Uttarakhand: Mussoorie

Contd. 
olivieri Lepeletier, 1825:448

Sphecodes collaris Spinola, 1843: 137

Sphecodes hispanicus var abyssinicus Sichel, 1865:447

Sphecodes ruficornis Sichel, 1865:440

Sphecodes punctulatus Sichel, 1865:443

Sphecodes subpunctulatus Sichel, 1865:445

Sphecodes rufithorax Morawitz, 1876: 225

Sphecodes verticalis Hagens, 1882:219

Sphecodes desertus Nurse, 1903:540

Sphecodes chionospilus Cockerell, 1911: 217

Sphecodes chionospilus var sanguinatus Cockerell, 1911: 217

Sphecodes tenuis Meyer, 1919: 121

Sphecodes olivieri var niveatus Meyer, 1925: $4 \quad$ Gujarat: Deesa

17 perplexus Nurse 1903

Sphecodes perplexus Nurse 1903: 540

shillongensis Blüthgen, 1927

Sphecodes shillongensis Blüthgen, 1927: 95

sikkimensis Blüthgen, 1927

Sphecodes sikkimensis Blüthgen, 1927: 54

simlaellus Blüthgen, 1927

Sphecodes simlaellus Blüthgen, 1927: 46

tantalus Nurse, 1903

Sphecodes tantalus Nurse, 1903: 539

turneri Cockerell, 1916

Sphecodes turneri Cockerell, 1916: 430
Jammu and Kashmir

Assam

Meghalaya

Sikkim

Himachal Pradesh: Simla

Jammu \& Kashmir: Kashmir, Ladia,

Assam

\section{Redescription of species Sphecodes crassicornis Smith, 1879: 28 \\ Male}

Color: Head and thorax black; abdomen ferruginous; pale white pubescence; apical fascia of tergum hyaline; Pilosity: White silky pubescence on paraocular, pronotum, around pronotal lobe, post occiput, metanotum, frons, alveolar space and supraclypeus; fine silky pale white pubescence appressed on anterior surface of lower pronotum, forecoxa, and posterior surface of propodeum; Short fine silky sparse plumose on mesepimeron; long sparse hairs on metanotum; dorsolateral propodeum with long, slightly dense silky pubescence; scattered hairs on anterior surface of T1; T3 to T6 with same kind of scattered hairs except apex; short fine sparse hairs on S1to S6 comparatively sparser than tergum. Sculpture: Small irregular, dense punctures on head; Punctures on vertex denser than in scutum with slightly elevated interspace (Plate.1c); supraclypeus elevated mid dorsally; frontal carina present; Punctures on clypeus slightly wider than supraclypeus and rest of the head; Small rounded uniformly dense punctures sparse on scutum (Fig.1a); same kind of punctures slightly wider on scutellum; Small wide punctures with smooth interspace on scutellum; metanotum with fine irregular dense punctures; dorsolateral and basal propodeum with strong sculpturing irregular reticulation; posterior margin irregularly defined; small wider punctures on posterior surface and strongly carinated at basolaterad; Tiny shallow punctures sparsely present on T1 (Fig. 1b, 1c); T2 with same punctures except marginal zone; shallow dense punctures on S2 to S5.

General: Male much smaller, $6.25 \pm 0.5 \mathrm{~mm}$; Body long slender, cylindrical; Head small, 1.16x as broad as long; antenna long, dorsolaterally flattened and reaches the scutellum; antennal flagellum stout; first flagellomere next to ring segment is small and slightly extend outwards; surface not smooth; clypeus $2.03 \mathrm{x}$ as long as clypeoantennal distance; compound eye $0.62 \mathrm{x}$ as long as head; upper interocular distance $0.78 \mathrm{x}$ as long as lower interocular; Alveolar distance $0.42 \mathrm{x}$ as long as antennocellar distance; Inter ocellar distance $0.78 \mathrm{x}$ as long as ocello-ocular distance; Scape 3.36x as long as pedicel; Flagellum $7 \mathrm{x}$ as long as scape; F1 $0.66 \mathrm{x}$ as long as pedicel and F2 $2.4 \mathrm{x}$ as long as pedicel;

Thorax: longer, almost rectangular; femur $5.2 \mathrm{x}$ as long as trochanter; hind tibia $0.79 \mathrm{x}$ as long as femur; basitarsi $0.66 \mathrm{x}$ as long as hind tibia; forewing length $4.75 \mathrm{x} \pm 0.05 \mathrm{~mm}$; Hindwing length $3.65 \pm 0.05 \mathrm{~mm}$; Tegulae $2 \mathrm{x}$ as long as broad; forewing $2.96 \mathrm{x}$ as long as broad; Hindwing 2.88x as long as broad; forewing $1.31 \mathrm{x}$ as long as and $1.28 \mathrm{x}$ as broad as hindwing; stigma $3.13 \mathrm{x}$ as long as broad, $4.54 \mathrm{x}$ as long as prestigma; prestigma $1.35 \mathrm{x}$ as long as broad; marginal cell $2.06 \mathrm{x}$ as long as free marginal cell and $1.42 \mathrm{x}$ as long as marginal cell length beyond stigma; marginal cell 

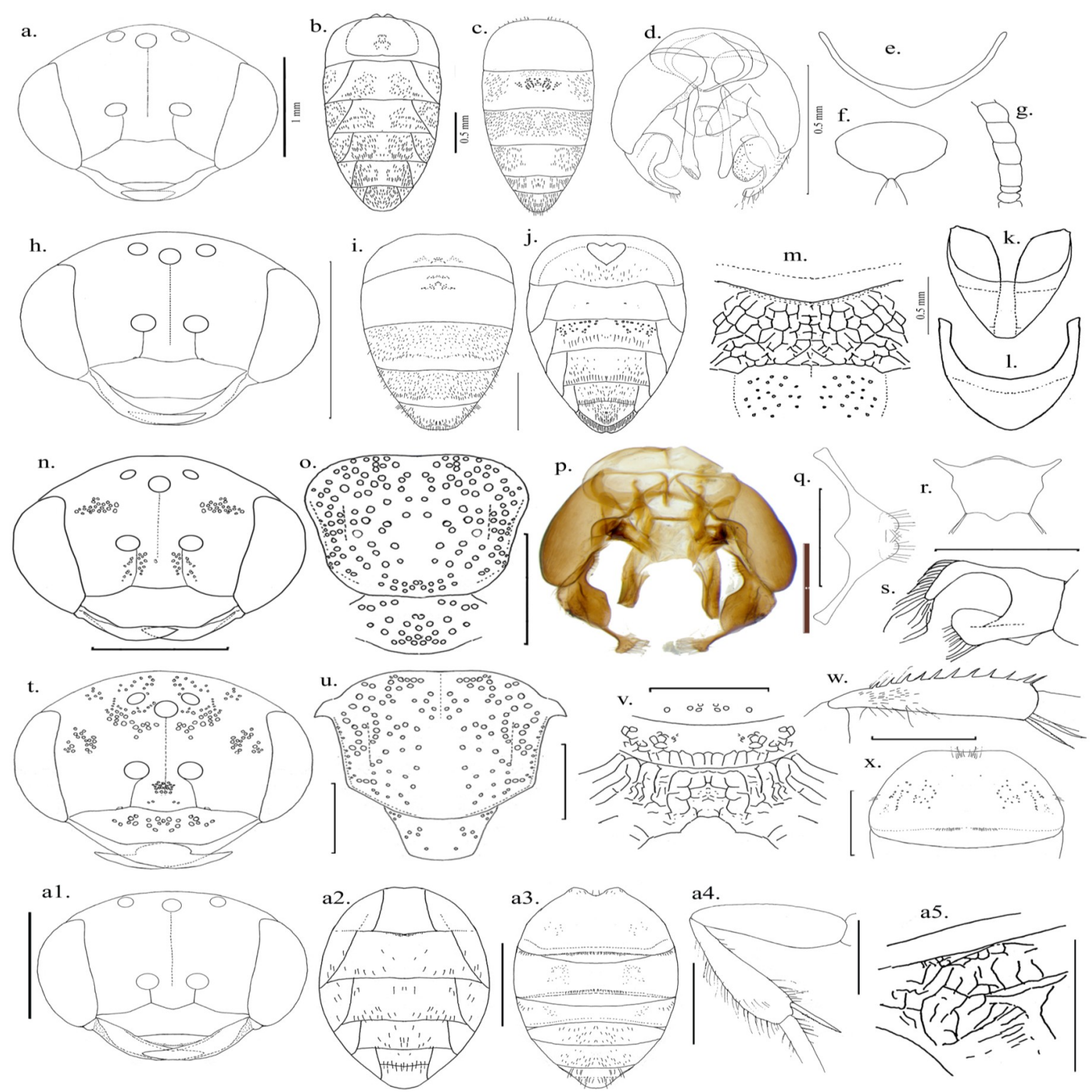

Fig. 1. (a to g) S. crassicornis $\delta^{\lambda}: a$, head; $b \& c$, abdomen ventral \& dorsal view; d, genitalia dorsal \& vental view. e, S7; $f$ S8; $g$, pedicel flagellomere (F1-F5); ( $h$ to $m$ ) $\rightarrow$ : $h$, head; $i \& j$, abdomen ventra \& dorsal view; $k$, propodium dorsal \& posterior view. l, T6;

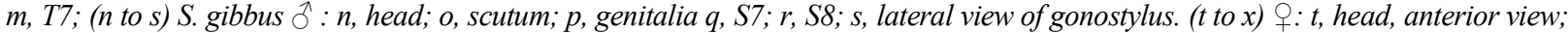
$u$, scutum \& sculellum; v, propodeum posterior view; w, hind tibia; $x$, T1. (a1 to a5) S. irridipennis 9 : al, head; a2 \& a3, abdomen dorsal and ventral viev; a4, hind femur \& tibia; a5, latero posterior view of propodeum.

$5.21 \mathrm{x}$ as long as broad; scutum $0.85 \mathrm{x}$ as long as broad at the anterior and $1.02 \mathrm{x}$ as long as broad at the posterior; scutum $2.93 \mathrm{x}$ as long as scutellum; scutellum $1.6 \mathrm{x}$ as long as metanotum; scutellum $1.92 \mathrm{x}$ as broad as long; Propodeum: metanotum $0.62 \mathrm{x}$ as long as propodeum; posterior surface $0.53 \mathrm{x}$ as broad as the length of propodeum; basal propodeum reticultated strongly and longer than metanotum; posterior surface slightly depressed inward; posterior surface with sparse short plumose; punctures irregular, slightly deep and scattered; Basal area of propodeum longer than metanotum; Tergum: simple, seems to be glabrous; T2 with transverse apparent depression near graduli;

Male genitalia: Oval, broad at middle, $1.12 \mathrm{x}$ as long as broad; laterally $0.56 \mathrm{x}$ as high as long; Gonobase $1.47 \mathrm{x}$ as broad as long; genital foramen broad equally as broad as long; Gonocoxite long, broad at base and $2.26 \mathrm{x}$ as long as broad; gonocoxite $0.32 \mathrm{x}$ as broad as the width of genitalia; penis valve slightly broader at apex and 10x as long as broad; gonostylus shorter; dorsal lobe of gonostylus $0.78 \mathrm{x}$ as broad as long where as ventral lobe $0.56 \mathrm{x}$ as broad as long; dorsal lobe parallel and elongated at apex with long hairs; the hairs $0.56 \mathrm{x}$ as long as dorsal lobe; ventral lobe broad oval; 

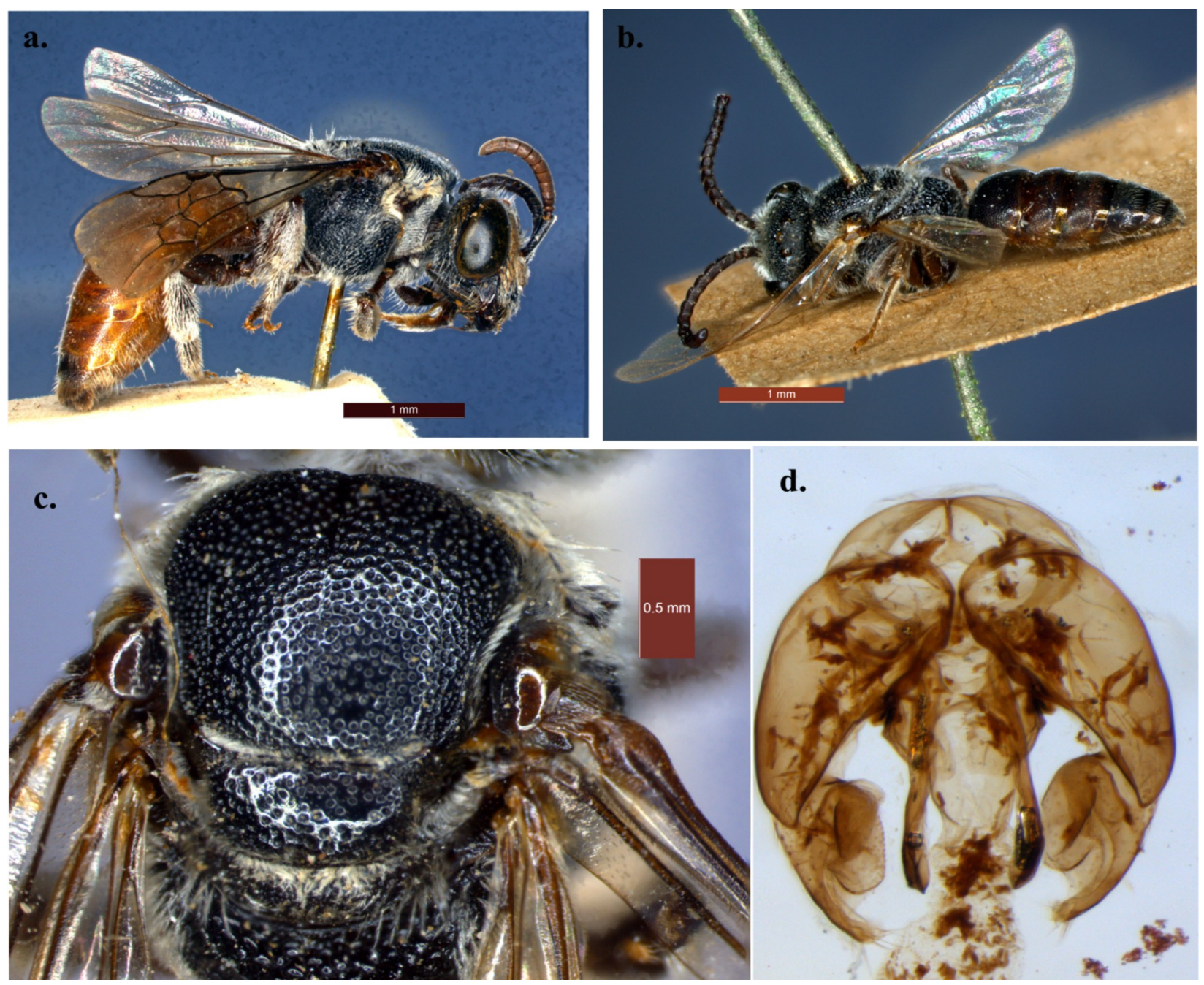

Plate 1. (a-d) S. crassicornis a\&b lateral hebitat of female \& male; c, dorcal view of thorax; $d$. dorsal and ventral view of male ganitalia.

Volsella longer, 0.37x as broad as long (Fig.1d; Plate. 1d);

Measurements: Body length $6.25 \pm 0.5 \mathrm{~mm}$; forewing length $4.75 \mathrm{x} \pm 0.05 \mathrm{~mm}$; Hindwing length $3.65 \pm 0.05$ $\mathrm{mm}$;

\section{Female}

Color: Body black; antenna, eyes and legs dark brown; abdomen ferruginous; apical fascia of tergum hyaline; Pilosity: Pale white dense plumose on frons, supraclypeus, paraocular alveolar area, pronotum, pronotal lobe and metanotum; fine plumose scattered on clypeus, hypostoma, occiput and vertex; Fine silky appressed pubescence on anterior surface of lower pronotum, forecoxa, mesepimeron, lateral and posterior surface of propodeum; pale white silky pubescence on along the lateral and posterior margin of propodeum; short fine silky plumose sparse on mesepimeron and post occiput; long sparse hairs on lateral metanotum; long silky dense pubescence on dorsolateral propodeum; short white plumose scattered on anterior surface of $\mathrm{T} 1$ and $\mathrm{T} 2$; short tiny sparse fine hairs on T2-T4; hairs comparatively longer on T5; fine slightly dense hairs on $\mathrm{S} 1$; short fine sparse hairs (sparser than tergum)on S2 to S4; long dense hairs at the apex of S5; Sculpture: Small irregular dense punctures on head; small and larger punctures sparsely present on scutum, the punctures wider on posterior scutum; small punctures scattered on scutellum with smooth interspace; punctures on vertex are denser and stronger than in scutum; supraclypeus strongly elevated; frontal line irregular; punctures on clypeus slightly wider than in supraclypeus and rest of the head; the scrobe of mesepimeron with areolated sculptures; small rounded dense punctures on scutum; punctures slightly dense near parapsidal lines fine irregular dense punctures on metanotum; strong irregular areolate on basal propodeum; posterior margin irregularly defined; small wider punctures on posterior surface; basolateral margin strongly carinated; tiny shallow sparse punctures on T1-T2 (Fig.1i, 1m); no punctures 

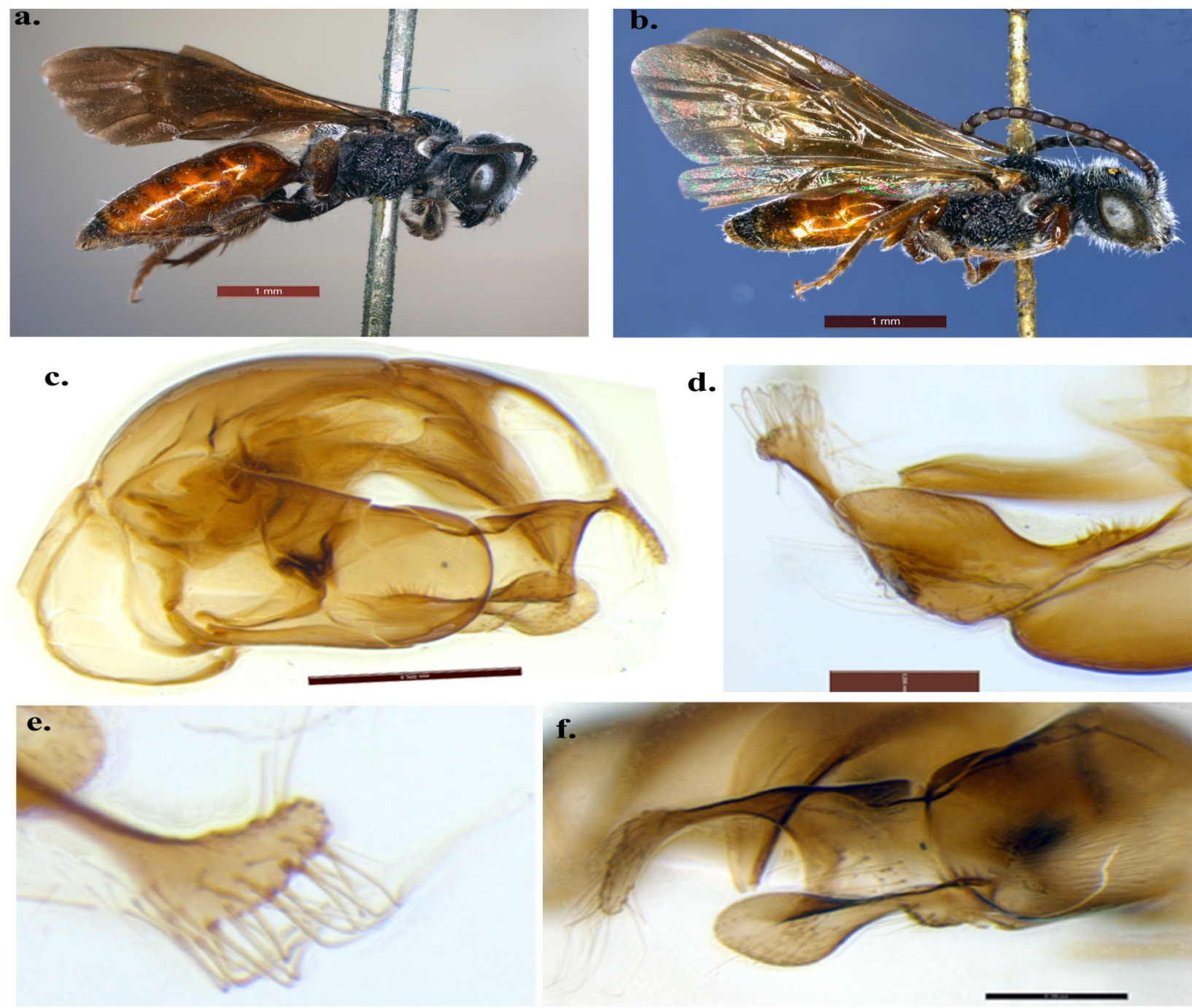

Plate 2. (a-f). S. gibbus a\&b lateral habitat of female \& male; c. genitalia lateral view; $d$, gonostylus dorsal view; e, apex of gonostylus; f, lateral view of gonostylus.

on marginal zone of T2; shallow sparse punctures on S2 to S5 (punctures larger than in tergum) (Fig. 1j);

General: Head 1.37x as broad as long; short alveolar distance; long scape; pedicel and F1 small; supraclypeus strongly convex; clypeus $1.5 \mathrm{x}$ as long as clypeoantennal distance; compound eye $0.66 \mathrm{x}$ as long as head; upper interocular $1.15 \mathrm{x}$ as long as lower interocular distance; frontal carina strong near alveolar space; clypeus elevated transversly in the middle and lateral apex bent down; mandible long; alveolar distance $0.28 \mathrm{x}$ as long as antennocellar distance; inter ocellar distance $0.61 \mathrm{x}$ as long as ocello ocular distance; scape $7.85 \mathrm{x}$ as long as pedicel; flagellum $2.14 \mathrm{x}$ as long as scape; F1 2.1x as long as pedicel; Thorax: basitarsi $0.65 \mathrm{x}$ as long as hind tibia; tegulae $1.91 \mathrm{x}$ as long as broad; forewing $2.91 \mathrm{x}$ as long as broad; Hindwing $3.50 \mathrm{x}$ as long as broad; forewing $1.29 \mathrm{x}$ as long as and $1.55 \mathrm{x}$ as broad as hindwing; stigma $3.46 \mathrm{x}$ as long as broad, $4.5 \mathrm{x}$ as long as prestigma; prestigma $1.66 \mathrm{x}$ as long as broad; marginal cell $1.70 \mathrm{x}$ as long as free marginal cell and $1.15 \mathrm{x}$ as long as marginal cell length beyond stigma; marginal cell $3.71 \mathrm{x}$ as long as broad; scutum $0.84 \mathrm{x}$ as long as broad at the anterior and $1.09 \mathrm{x}$ as long as broad at the posterior; scutum $3.08 \mathrm{x}$ as long as scutellum; scutellum $1.53 \mathrm{x}$ as long as metanotum; Propodeum: basal propodeum longer than metanotum; metanotum $0.75 \mathrm{x}$ as long as propodeum; posterior surface slightly depressed; Abdomen: seems to be glabrous but with sparse tiny pale brown hairs; transverse depression near graduli on $\mathrm{T} 2$;

Measurements: Body length $9.15 \mathrm{~mm}$; forewing length $7.0 \mathrm{~mm}$; hindwing length $5.4 \mathrm{~mm}$

Materials examined: Male, Coimbatore, Tamil Nadu, April, 1915, C.R. Dutt, P. Bluthgen det. H-6341; Female, H-6339, (syn: sodalist smith, abuense Nurse, des. Bluthgen) banhar, , behar, H. Inglis Coll., 16.5.19; Distribution: Tamil Nadu: Coimbatore; West Bengal: Kolkata 

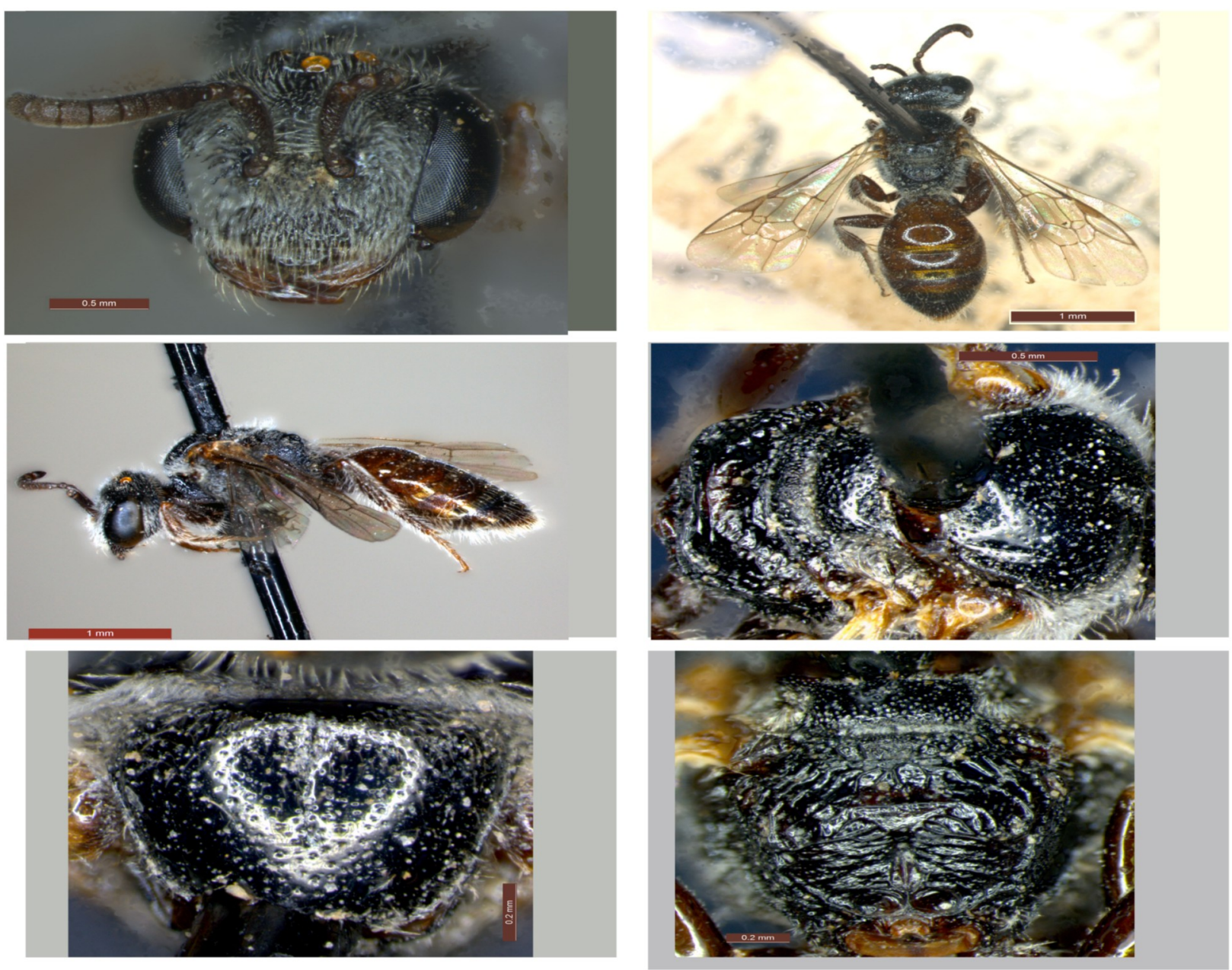

Plate 3. (a-f) irridipennis $ᄋ$ : $a$, head anterior view; $b \& c$, dorsal \& lateral habitat; $d$, thorax dorsal view; e, scutum; Propodeum posterior view.

\section{Spehcodes gibbus (Linnaeus 1785:571) \\ Male}

Color: Body ferruginous; head, scape and thorax black; legs, mandible, flagellum and compound eye dark brown; wings fuscous and hyaline apex; S1 brownish red; S2 ferruginous; Pilosity: Generally fine hairs scattered all over the body; tiny hairs sparse on flagellum and dense at base of each flagellomere; thick pubescence on paraocular area; sparse, short hairs on clypeus, scape, frons and vertex; tiny dense white hairs on pronotum and pronotal lobe; fine long amber hairs scattered on mesepisternum, sparse on femur, tibia and forebasitarsi; fine long hairs scattered on posterior hind coxa, trochanter, femur, mesepisternum, anteclypeal margin and mandible; long sparse hairs on outer hind tibia; anterior hind coxa, trochanter and femur glabrous; short plumose scattered on anterior surface of $\mathrm{T} 1$; short tiny hairs scattered at laterad and premarginal area of T1 to T3; Sculpture: small dense strong punctures on head; small punctures on supraclypeus and ocular margin; punctures scattered on clypeus, and vertex near mid ocelli; punctures denser on paraocular area; scattered shallow punctures on occiput; transverse discontinuous carina on post occipital region; large rounded punctures sparsely on scutum and scutellum; interspace smooth; tiny punctures scattered on scape; irregular strong dense punctures on metanotum; basal propodeum strongly reticulated; posterior surface of propodeum with defined marginal carina; large strong punctures scattered on $\mathrm{T} 1$ mixed along with tiny and small punctures; marginal zone glossy; punctures on $\mathrm{T} 1$ comparatively stronger than female; marginal disc broad at the middle; premarginal line strong at laterad on T3 than T2, T1; tiny dot like punctures on T1 to T3 (Fig. 1n, 1o).

General structure: Head $1.15 \mathrm{x}$ as broad as long; clypeus $1.13 \mathrm{x}$ as long as clypeoantennal distance; compound eye $0.62 \mathrm{x}$ as long as head; antenna dorsoventrally flattened and reaches beyond scutum; scape $3.3 \mathrm{x}$ as long as pedicel; pedicel $0.27 \mathrm{x}$ as long as F11; scape $0.03 \mathrm{x}$ as long as flagellum; F1 equally long as pedicel; upper interocular $1.2 \mathrm{x}$ as long as lower interocular distance; alveolar distance $0.74 \mathrm{x}$ as long as antennocellar distance; inter ocellar distance $1.41 \mathrm{x}$ as 
long as ocello ocular distance; hind legs are slender; Thorax: scutum $0.88 \mathrm{x}$ as long as broad at the anterior and $1.05 \mathrm{x}$ as long as broad at the posterior; scutum 2.46x as long as scutellum; metanotum oblong; scutellum 1.6x as long as metanotum; Hindcoxa longer than other coxae; femur $4.6 \mathrm{x}$ as long as trochanter; hind tibia $1.29 \mathrm{x}$ as long as femur; basitarsi $0.50 \mathrm{x}$ as long as hind tibia; wings are fuscous and their apex hyaline; legs are slender compared to body; tegula small, oval and $1.5 \mathrm{x}$ as long as broad; forewing $2.71 \mathrm{x}$ as long as broad; forewing $1.25 \mathrm{x}$ as long as and $1.9 \mathrm{x}$ as broad as hindwing; stigma $2.43 \mathrm{x}$ as long as broad, $4.18 \mathrm{x}$ as long as prestigma; marginal cell $1.91 \mathrm{x}$ as long as free marginal cell and $2.87 \mathrm{x}$ as long as marginal cell length beyond stigma; marginal cell $4.1 \mathrm{x}$ as long as broad; posterior surface of propodeum depressed in the middle; metanotum $1.51 \mathrm{x}$ as long as propodeum; abdomen long cylindrical; Sternum: S2 transparent amber glossy; graduli strong and not straight depression below; S3 blackish transparent short scattered fine hairs; S4, S5 sparse short; S5 1.89x as long as broad; S6, $1.68 \mathrm{x}$ as broad as long and mid disc broad, with short, sparse hairs both sides of middle lateral; S7 transverse, wider and broad at the middle; $3.5 \mathrm{x}$ as long as broad; mid apical lobes with sparse fine erect hairs; S8 0.88x as broad as long with short lateral apodeme; mid apical lobe simple; lateral apex with small bunch of long fine hairs; disc is broad;

Measurements: Body length $8.0 \pm 0.05 \mathrm{~mm}$; forewing length $6.56 \pm 0.03 \mathrm{~mm}$; hindwing length $2.60 \pm 0.03$ $\mathrm{mm}$;

Male genitalia:, Oval, greatly convex and broader at middle; $0.98 \mathrm{x}$ as long as broad; laterally $0.61 \mathrm{x}$ as high as long; gonocoxite long oval and $0.20 \mathrm{x}$ as broad as the length of genitalia; gonobase $1.9 \mathrm{x}$ as broad as long; gonostylus laterally broader and concave; dorsal lobe $1.45 \mathrm{x}$ as long as broad and the apex $0.5 \mathrm{x}$ as broad as long; the dorsal lobe with deep semicircular cleft at preapex; apex narrow towards down with long bent hairs; ventral lobe $2.6 \mathrm{x}$ as long as broad and $3.6 \mathrm{x}$ as broad as dorsal lobe; the ventral lobe smaller than dorsal lobe, broader at apex with short, fine hairs and few strong hairs at their base; penis valve smaller, simple with apical and midlateral tooth; penis valve $7.4 \mathrm{x}$ as long as broad; hairs of dorsal lobe $0.93 \mathrm{x}$ as long as their apex (Fig. 1p, 1s; Plate. 2c-2f.);

\section{Female}

Females differ with males in size, antenna, abdomen, hind tibia, size of the punctures and mandible; Head: punctures same as present in male except the small punctures are slightly dense at the mid clypeus; antenna small, without tiny hair bands at the of base each flagellomere; punctures on the scutum and scutellum are smaller than in male and widely scattered; few smaller punctures scattered on scutellum; mandible longer than male; posterior margin of outer hind tibia with a row of tooth along the margin, angulated and directed downward; hind tibia slightly broadened at apex; abdomen broader at the middle; T1 and T2 completely ferrugenous; tiny small punctures scattered on T1-T2 and not as strong as in males (Fig. 1x); marginal zone transparent glossy, through this antecostal suture visible and row of fine tiny hairs visible; Metanotum almost rectangular with slightly angled posterior margin (Fig. 1t- 1v).

General structure: head $1.31 \mathrm{x}$ as broad as long; clypeus $1.31 \mathrm{x}$ as long as clypeoantennal distance; compound eye $0.61 \mathrm{x}$ as long as head; upper interocular $0.97 \mathrm{x}$ as long as lower interocular distance; Alveolar distance $0.55 \mathrm{x}$ as long as antennocellar distance; inter ocellar distance $0.53 \mathrm{x}$ as long as ocello ocular distance; scape $2.61 \mathrm{x}$ as long as pedicel; flagellum $2.27 \mathrm{x}$ as long as scape; F1 $0.44 \mathrm{x}$ as long as pedicel; thorax: femur $1.23 \mathrm{x}$ as long as trochanter; hind tibia $1.06 \mathrm{x}$ as long as femur; basitarsi $1.58 \mathrm{x}$ as long as hind tibia; tegulae $2 \mathrm{x}$ as long as broad; forewing $2.85 \mathrm{x}$ as long as broad; Hindwing $2.84 \mathrm{x}$ as long as broad; forewing $1.28 \mathrm{x}$ as long as and $1.31 \mathrm{x}$ as broad as hindwing; stigma $0.31 \mathrm{x}$ as long as broad, $3.41 \mathrm{x}$ as long as prestigma; prestigma $2.4 \mathrm{x}$ as long as broad; marginal cell $1.84 \mathrm{x}$ as long as free marginal cell and $1.27 \mathrm{x}$ as long as marginal cell length beyond stigma; marginal cell $0.24 \mathrm{x}$ as long as broad; scutum $0.75 \mathrm{x}$ as long as broad at the anterior and $0.95 \mathrm{x}$ as long as broad at the posterior; scutum $2.42 \mathrm{x}$ as long as scutellum; scutellum $1.61 \mathrm{x}$ as long as metanotum; Propodeum: scutellum $1.6 \mathrm{x}$ as broad as long; propodeum $1.6 \mathrm{x}$ as long as metanotum; posterior surface $2.52 \mathrm{x}$ as broad as the length of propodeum; scutellum $0.64 \mathrm{x}$ as long as broad;

Measurements: Body length $12.2 \pm 0.03 \mathrm{~mm}$; forewing length $9.9 \pm 0.03 \mathrm{~mm}$; hindwing length $3.47 \pm$ $0.03 \mathrm{~mm}$;

Materials examined: Male, H-6337, Kashmir, 5200ft, Srinagar, Jun-Aug, 1923, P Bluthgen, det, , Dutt Coll.; Male, H-6336, Kashmir, 5200ft, Srinagar, Jun-Aug, 1923, P Bluthgen, det, , Dutt Coll.; Female, H-6333, Kashmir, 5200ft, Srinagar, Jun-Aug, 1923, P Bluthgen, det, , Fletcher coll.; Male, H-6334, Kashmir, 5200ft, Srinagar, Jun-Aug, 1923, P Bluthgen, det, Dutt Coll.;

Distribution: India: Kashmir;

Sphecodes iridipennis Smith, 1879: 27

Female (Fig 1. a1 to a5)

Color: Body black; metasoma ferrugenous; dark brown antenna, sternum; Pilosity: sparse fine, white pubescence on paraocular, supraclypeus, pronotum, pronotal lobe, frons and very sparse on clypeus; fine, scattered plumose on vertex and occiput; pale white hairs, sparsely on anterior surface of pronotum; tiny scattered hairs on scutum; fine pale short hairs on anterior margin of metanotum; dorsal and lateral propodeum glabrous; long white silky sparse subdecumbent hairs on femur, tibia and basitarsi of all the legs; short tiny, fine hairs on mid premarginal line; T1 glossy, 
smooth, and tiny hairs scattered on anterior surface; long scattered hairs on marginal zones of all the tegum; slightly long very fine scattered hairs on lateral apex of S1 to S3; same kind of hairs sparsely present on S4 to S6; Sculpture: fine dense shallow punctures on paraocular, vertex, clypeus, supraclypeus and occiput ; frontal carina irregular strong and reaches upto interantennal area; shallow rounded punctures on scutum and scutellum and sparser than in head (Plate 3e); supraclypeus slightly elevated;-- irregular sculptures on metanotum; mid and forefemur glabrous; basal propodeum strongly reticulted and carinated strongly at posterior margin; interspace smooth and shiny; transverse carina towards the pit on posterior propodeum; small shallow punctures scattered on T1; small shallow sparse punctures on $\mathrm{T} 2$ to $\mathrm{T} 5$; few shallow sparse punctures on midlateral $\mathrm{S} 1$; the same kind of punctures laterally on $\mathrm{S} 2$ to $\mathrm{S} 5$; mesepimeron scrobe areolated (Fig. 1a1, 1a2);

General structure: Head: scape long; pedicel and F1 smaller; supraclypeus slightly elevated; frontal carina well defined; clypeus short and transversly elevated in the middle; mandible long, strong preapical tooth; scape longer; head 1.30x as broad as long; clypeus $1.2 \mathrm{x}$ as long as clypeoantennal distance; compound eye $0.73 \mathrm{x}$ as long as head; upper interocular $0.91 \mathrm{x}$ as long as lower interocular distance; alveolar distance $0.5 \mathrm{x}$ as long as antennocellar distance; inter ocellar distance $0.93 \mathrm{x}$ as long as ocello ocular distance; scape $5 \mathrm{x}$ as long as pedicel; flagellum $2.13 \mathrm{x}$ as long as scape; F1 $0.75 \mathrm{x}$ as long as pedicel (Plate. $3 \mathrm{a}$ ); Thorax: metanotum smaller than scutellum; hind femur slightly broader at base; hind tibia broader at apex; tibial spur long narrow towards apex and lower margin finely serrated near base; Femur $2 \mathrm{x}$ as long as trochanter; hind tibia $1.02 \mathrm{x}$ as long as femur; basitarsi $0.65 \mathrm{x}$ as long as hind tibia; Tegulae $1.4 \mathrm{x}$ as long as broad; forewing $3.26 \mathrm{x}$ as long as broad; Hindwing $3.37 \mathrm{x}$ as long as broad; forewing $1.30 \mathrm{x}$ as long as and $1.35 \mathrm{x}$ as broad as hindwing; Stigma $3 \mathrm{x}$ as long as broad marginal cell, $1.50 \mathrm{x}$ as long as free marginal cell and $1.22 \mathrm{x}$ as long as marginal cell length beyond stigma; marginal cell $3.5 x$ as long as broad; scutum $0.82 \mathrm{x}$ as long as broad at the anterior and $1.20 \mathrm{x}$ as long as broad at the posterior; scutum $2.61 \mathrm{x}$ as long as scutellum; scutellum $1.63 \mathrm{x}$ as long as metanotum; scutellum 1.66x as broad as long (Plate. 3d); Propodeum: basal propodeum depressed in the middle (Plate. 3f); posterior surface slightly depressed; metanotum $0.68 \mathrm{x}$ as long as propodeum; posterior surface $3.18 \mathrm{x}$ as broad as the length of propodeum (Fig. 1a5); Abdomen: T1anterior surface slightly flattened; T3 marginal zone slightly longer in the middle than $\mathrm{T} 1$;

Measurements: Body length $5.10 \pm 0.30 \mathrm{~mm}$; forewing length $4.41 \pm 0.03 \mathrm{~mm}$; hindwing length $3.37 \pm 0.03 \mathrm{~mm}$;

Materials examined: Male/Female, H-6346, GR Dutt, Chapra, Bengal, Mackenzie;

Distribution: Bihar: Chapra.

\section{DISCUSSION}

In the present study, the existing information on the three species viz., $S$. crassicornis, $S$. gibbus, S.iridipennis was supplemented with additional description, illustrations and morphometric ratios of important taxonomic features including male genitalia. Description of pilosity, sculpture and male genitalia focused in this study is the recent trend in bee taxonomy used by bee specialists. It is difficult to recognize species of genus Sphecodes as they are cleptoparasitic and obviously do not possess much specialized features. The male genitalia and sculpture of mesosoma are the well-built features for identification of species. Generally the females are much bigger and males are slender with longer antenna. All the three species generally varied in size and the type of punctures on mesosoma viz., small, dense punctures in S. crassicornis, large sparse punctures in $S$. gibbus and shallow punctures in $S$. irridipennis; the density of punctures slightly vary between the sex, for example, the punctures on scutum of male S. gibbus is denser than in female. Likewise it was observed in S. crassicornis. As far as the male genitalia is concerned, wide variation was observed on gonostylus. The dorsal lobe of gonostylus with deep semicircular cleft was observed in $S$. gibbus while it is parallel in case of $S$. crassicornis.

The redescription is necessary in the context of increasing attention on bee diversity and advent of new species. It needs in depth studies on the morphology to identify key feature which helps in differentiating relatively closer species. This study may be stepping stone for identification of all the Indian species under genus Sphecodes Latreille 1804.

\section{Conclusion}

The taxonomy of genus Sphecodes has been inadequately worked out in India during the past. The earlier descriptions lacked illustrations especially those of male genitalia and photographs. The present study bridges the gap in existing knowledge.. The check list also has been prepared and it will be useful in future taxonomic studies.

\section{ACKNOWLEDGEMENTS}

The first author acknowledges the Director \& Dean, IARI, New Delhi for providing fellowship during the course of study.

\section{REFERENCES}

Ascher, J. S. and J. Pickering (2014). Discover life bee species guide and world checklist (Hymenoptera : Apoidea: Anthophila), http://www.discoverlife.org/ $\mathrm{mp} / 20 \mathrm{q}$ ?guide=Apoidea_species

Bingham, C. T. (1897). The Fauna of British India Including Ceylon and Burma, Hymenoptera, Vol. I. Wasps and 
Bees. Xxix +577 p., 4 pls. London: Taylor and Francis. (Reprinted, 1975, New Delhi: Today and Tommorrow's)

Bingham, C. T. (1898). On some new species of Indian Hymenoptera volume.12 part I. Journal of the Bombay Natural History Society, 12: 1-234

Bluthgen, P (1924). Zur Systematik der Bienengattung Sphecodes Latr. II. Deutsche Entomologische Zeitschrift issue, 6: 456-516

Blüthgen, P. (1927). Beiträge zur Systematik der Bienengattung Sphecodes Latr. III. Zoologische Jahrbücher, Abteilungfur Systamatik, Geographie und Biologie der Tiere, 53: 23-112

Blüthgen, P. (1933). Halictus Latr., Sphecodes Latr., Nomioides Schenck, p. 52-63 In: A. Nadig, sen. \& Jun. Contribution to the knowledge of the Hymenopteren fauna of Morocco and western Algeria. Part One: Apidae, Sphegidae, Vespidae. Annual reports of the nature of Research Society, 71:37-107

Cameron, P. 1896 (1897). Hymenoptera Orientalia, or Contributions to the knowledge of the Hymenoptera of the Oriental zoological region, Part V. Memoirs and Proceedings of the Manchester Literary and Philosophical Society, 41(2): 1-144

Cockerell, T. D. A. (1911). New and little known bees. Transactions of the American entomological Society, 37: 217-234.

Cockerell, T. D. A. (1938). Bees of the genus Sphecodes from the Belgian Congo. Revue de Zoologie et de Botanique aficans, 30: 327-329

Dalla Torre, C. G. de. (1896). Catalogus Hymenopterorum, Apidae (Anthophila). Leipzig: Engelmann.10 (viii): pp 643

Friese, H. (1909). Die Bienen Afrikas nach dem Stande unserer heutigen Kenntnisse, In L. Schultz, Zoologische und Anthropologische Ergebnisse einer Forschungsreiseim westlichen und zentralen südafrika ausgefuhrt in den Jahren 1903-1905, Jena: Fischer. Band 2, Lieferung 1, X Insecta (ser.3) (Jenaische Denkschrifen) Vol. 14: pp. $83-476$

Hagens, D. Von. (1882). Ueber die männlichen Genitalien der Bienen-Guttung Sphecodes. Deutsche Entomologische Zeitschrift 26: 209-228.

Illiger, K. (1806). William Kirbys Familien der Bienenartigen Insekten mit Zusätzen, Nachweisungen und Bemerkungen. Magazin für Insektenkunde 5:28-175

Kirby, W. (1802). Monographia Apum Angliae, Ipswich, U. K. Privately published Vol. 1\&2: pp 388.
Latereille, P. A. (1804). Tableau méthodique des insectes, In: Nouveau Dictionnaire d'Histoire Naturelle, Paris Déterville Vol. 24: pp.129-200

Lepeletier de Saint-Fargeau, A. (1841). Natural history of insects, Hymenoptera. Vol.2, Roret encyclopedic library, Paris. 2: pp 680

Linnaeus, C. (1758). Systema Naturae, The system of nature through the three kingdoms of nature, according to classes, Orders, genera, species, cum characters, differences, synonyms, locis. Holmiae: Salvii 1: $824 \mathrm{p}$

Meyer, R. (1919). Apidae, Sphecodinae I. Archiv für Naturgeschichte 85 A 1:79- 242

Meyer, R. (1925). Zur Bienengattung Sphecodes. Archiv für Naturgeschichte 90 A 12:1-12

Michener, Charles. D. (2007). The bees of the world, second edition. The Johns Hopkins University Press, Baltimore, Maryland 21218-4363. pp 953

Morawitz, F. (1876). Bienen (Mellifera). II. Andrenidae. In: FEDTSCHENKO, A. P: Reisen in Turkestan I. Izv. Imp. Obshch. Ljubit. Estest. Antrop. Etnog. Taf. 1-3. 21: 161-303

Nurse, C. G. (1903). New species of Indian Aculeate Hymenoptera. Annals and Magazine of Natural History, 11: 393-403, 511-526, 528-549

Robertson, C. (1903). Synopsis of Sphecodinae. Entomological News, 14: 103-107.

Sichel, J. 1865. Révision monographique, critique et synonymique du genre mellifera Sphecodes Latr. Annales de la Société Entomologique de France (4) 5: 397-466

Smith, F. (1845). Weibchen Männchen, England Zoologist 3: 1014

Smith, F. (1853). Catalogue of Insects in the Hymenopterous collections of the British Museum, Part I. Andrenidae and Apidae. Printed by order of the trustees, British Museum, London 1: $197 \mathrm{p}$

Smith, F. (1879). Description of new species of Hymenoptera in the collection of the British Museum. Printed by order of the trustees, British Museum, London. xxi: pp 240

Tsuneki, K. (1983). A contribution to the knowledge of Sphecodes Latreille of Japan (Hymenoptera, Halictidae). Special Publications of the Japan Hymenopterists Association, 26: 1-71

Verhoeff, C. (1890). Ein Beitrag zur deutschen Hymenopteren-Fauna. Entomologische Natchrichten 16: 321-335

Warncke, K. (1992). Die westpaläarktischen Arten der Bienengattung Sphecodes Latr. Bericht der Naturforschende Gesellschaft Augsburg, 52: 9-64 